\title{
EchoGéo
}

40 | 2017

Ressources urbaines (2)

\section{Ressources urbaines à la Frontera Norte}

\section{Aurélia Michel and Antonine Ribardière}

\section{(2) OpenEdition}

\section{Journals}

\section{Electronic version}

URL: https://journals.openedition.org/echogeo/14947

DOI: 10.4000/echogeo.14947

ISSN: 1963-1197

\section{Publisher}

Pôle de recherche pour l'organisation et la diffusion de l'information géographique (CNRS UMR 8586)

\section{Electronic reference}

Aurélia Michel and Antonine Ribardière, "Ressources urbaines à la Frontera Norte", EchoGéo [Online], 40 | 2017, Online since 30 June 2017, connection on 10 August 2021. URL: http://

journals.openedition.org/echogeo/14947 ; DOI: https://doi.org/10.4000/echogeo.14947

This text was automatically generated on 10 August 2021

EchoGéo est mis à disposition selon les termes de la licence Creative Commons Attribution - Pas d'Utilisation Commerciale - Pas de Modification 4.0 International (CC BY-NC-ND) 


\title{
Ressources urbaines à la Frontera Norte
}

\author{
Aurélia Michel and Antonine Ribardière
}

1 La ville d'Agua Prieta, dans l'État du Sonora au Mexique, a connu une croissance urbaine accélérée au cours des trente dernières années, typique des villes de la frontière avec les États-Unis ${ }^{1}$. L'industrie maquiladora ${ }^{2}$, dédiée à l'assemblage pour l'exportation, a constitué le moteur de la croissance. Alors que la ville compte à peine 20000 habitants en 1964 lors de l'installation de la première usine, la population augmente brutalement à partir de 1990, pour dépasser 60000 au début des années 2000 . En 2015, le municipe d'Agua Prieta compte plus de 80000 personnes.

2 Ce développement rapide, dû à la croissance d'un secteur d'activité bien spécifique, a-til produit des ressources urbaines, telles que nous essayons de les définir dans ce numéro (Michel, Ribardière, 2017) ? Comment la maquiladora oriente-t-elle la fabrique de la ville et l'accès aux ressources?

3 Agua Prieta, ville moyenne de la Frontera Norte entre les géants Mexicali et Ciudad Juárez, illustre de manière exemplaire l'articulation entre position frontalière, système des maquiladoras, migrations et production urbaine ${ }^{3}$. 


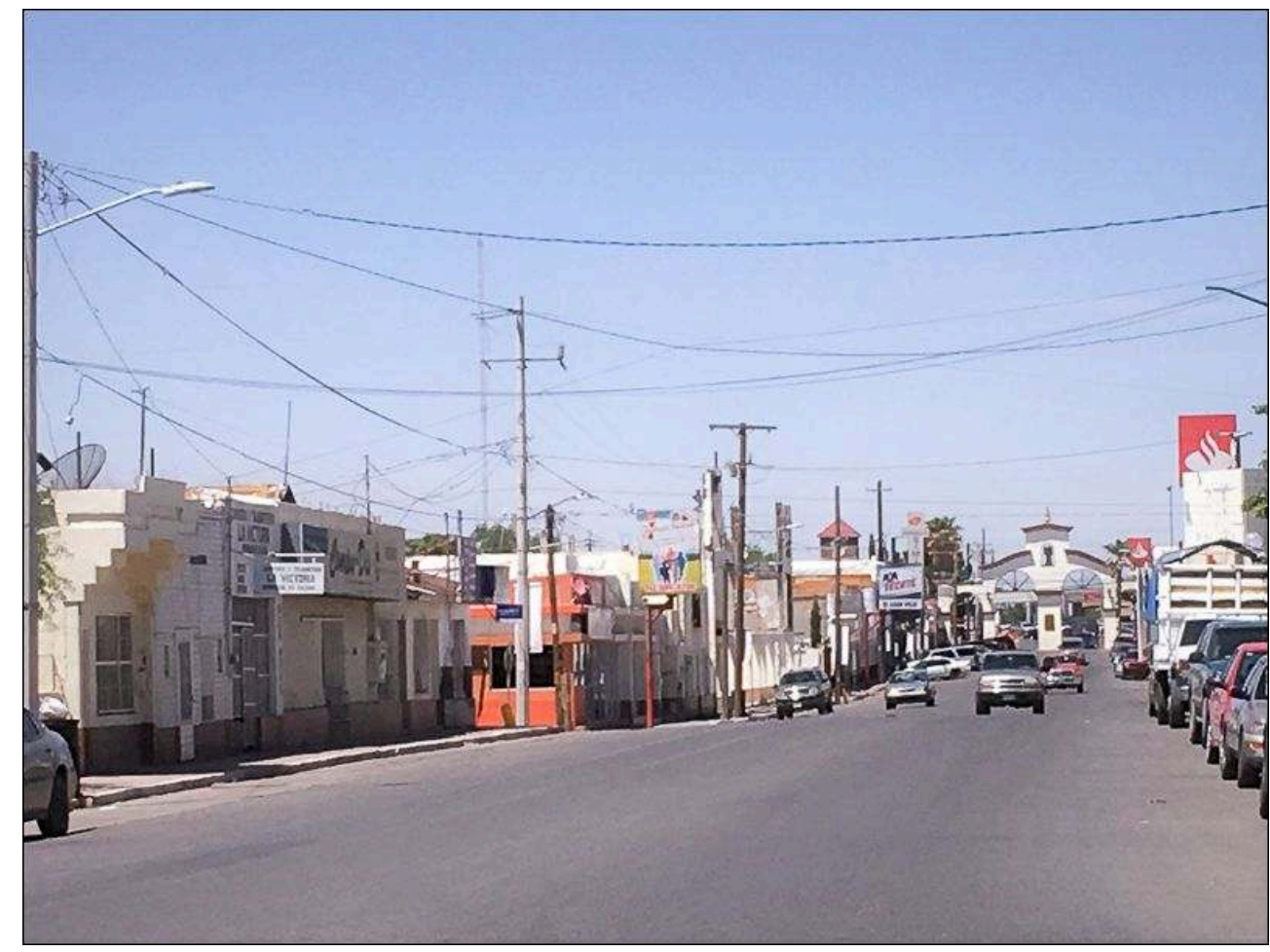

Il s'est développé à partir de la douane, ici au dernier plan. Les quatre premières rues parallèles à la frontière sont les plus cotées et les terrains peuvent valoir plus de 100000 dollars.

Auteurs : Aurélia Michel et Antonine Ribardière, 2017.

4 Fondée en 1899, la localité d'Agua Prieta est alors un point de passage du chemin de fer qui achemine le minerai de cuivre (provenant de gisements voisins, à Cananea et Nacosari) vers la fonderie de Douglas aux États-Unis (Arizona). L'installation d'une douane en 1918 consolide la localité. Mais c'est avec l'arrivée de la première maquiladora en 1964 que la ville prend véritablement son essor. Les usines d'assemblage se multiplient au cours des années 1970, comme tout le long de la bande frontalière où elles bénéficient d'avantages fiscaux. La ville compte jusqu'à 35 maquiladoras. Sur la période récente, la crise financière américaine de 2008 a infligé une régression sévère, mais l'activité industrielle s'est maintenue : aujourd'hui, 19 entreprises différentes ont installé une partie de leur chaîne de production à Agua Prieta. Malgré les incertitudes liées à l'arrivée à la présidence de Donald Trump, quatre entreprises ont tout de même choisi d'augmenter leur production en 2017: quatre nouveaux sites sont ainsi actuellement en construction. 
Illustration 2 - Les publicités pour le recrutement dans les usines sont omniprésentes dans la ville

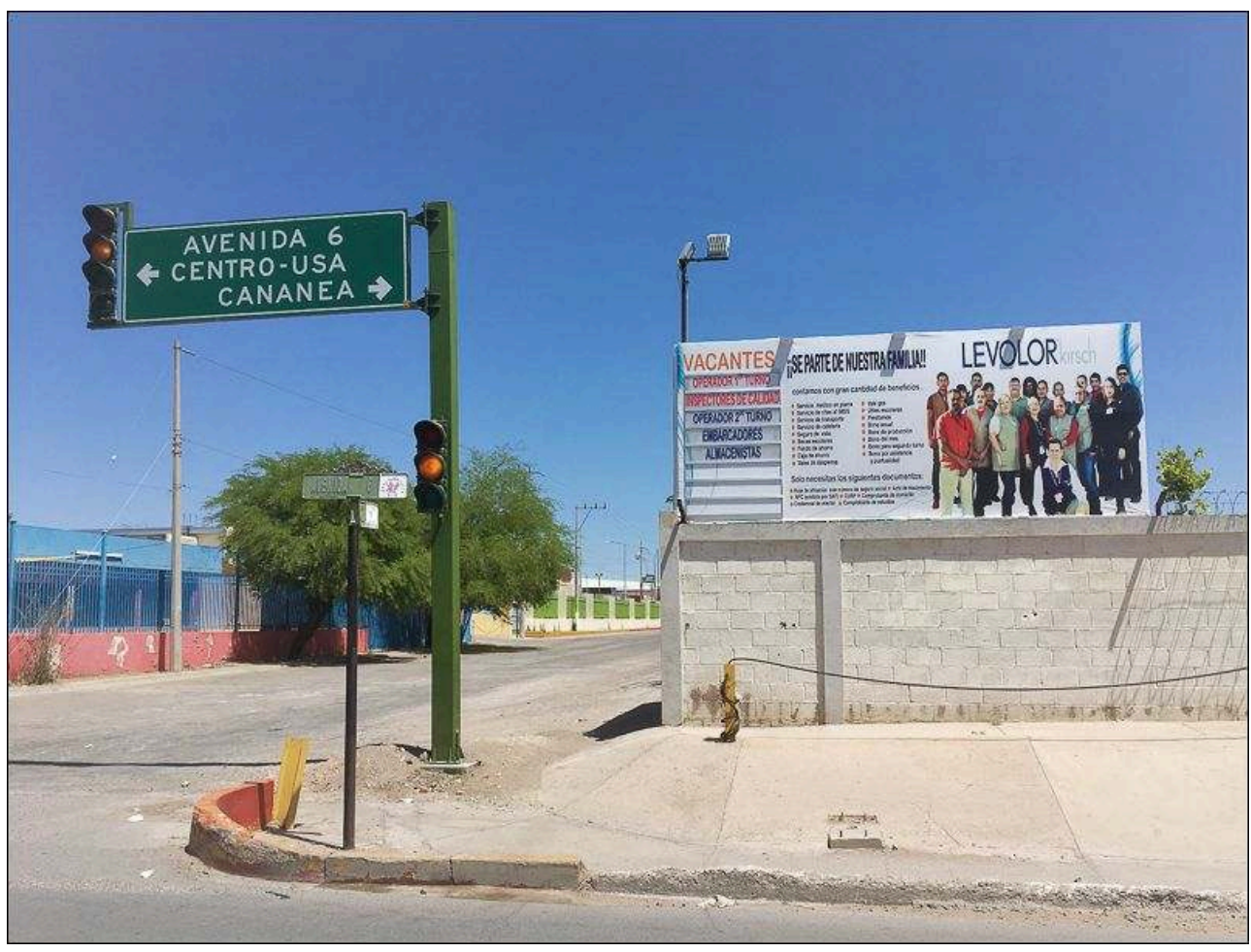

La proximité des États-Unis constitue l'autre facteur d'attractivité de la localité pour les migrants. Les entreprises sont fortement concurrentes pour le recrutement d'ouvriers et si elles proposent toutes à peu près le même salaire de base, elles cherchent à se distinguer sur les beneficios, avantages liés à l'emploi, ici détaillés (service médical en usine, service de prise de rendez-vous à l'hôpital de la sécurité sociale, service de transport, service de cafétéria, assurance civile, fonds d'épargne, caisse d'épargne, bons alimentaires, bons pour le gaz, fournitures scolaires, crédit, prime annuelle, prime de productivité, prime mensuelle, prime pour horaires décalés, prime pour présence et ponctualité).

Auteurs : Aurélia Michel et Antonine Ribardière, 2017

Cette deuxième photographie illustre l'articulation des différentes dimensions qui caractérise la situation à la Frontera Norte. Tout d'abord, les entreprises exploitent le différentiel de salaire considérable de part et d'autre de la frontière. En effet, le salaire de base pour un poste d'opérateur dans une maquiladora d'Agua Prieta en 2017 est d'environ 100 pesos par jour - soit 5 USD - alors que le salaire minimum horaire atteint 10 USD en Arizona. L'installation sur la frontière même réduit au maximum les coûts logistiques de l'accès au marché américain. Lorsqu'une entreprise s'installe, elle négocie conjointement la location de l'unité de production dans le parc industriel d'Agua Prieta et la location d'entrepôts dans la ville jumelle américaine de Douglas ; elle bénéficie des mesures d'exonération prévues par l'État fédéral mexicain pour les entreprises de montages destinées à l'exportation (réduction de la TVA à $11 \%$, au lieu de $19 \%)$.

6 Ensuite, la frontière marque plus généralement un différentiel de ressources, inégalement accessibles pour la population locale. Le statut administratif vis-à-vis de l'État américain (citoyen, frontalier, touriste ou interdit de traversée) détermine les conditions d'accès aux ressources convoitées des États-Unis et ce faisant, constitue un marqueur puissant de la hiérarchie sociale. On peut considérer qu'il y a trois catégories: les plus vieilles familles, qui possèdent le foncier à travers l'ejido ${ }^{4}$, ont généralement acquis la citoyenneté américaine depuis plusieurs générations, lorsque 
celle-ci était facilement attribuée avant le durcissement des lois migratoires et le programme Bracero des années 1960. Elles profitent ainsi de toutes les ressources du système éducatif et de santé des États-Unis. Après la citoyenneté complète étasunienne, un deuxième niveau d'accessibilité est offert par le visa Centri. Ce droit de passage permanent permet la circulation la plus fluide possible, avec des contrôles frontaliers réduits au minimum. Il est ainsi possible de déposer ses enfants tous les matins dans une école à Douglas ou de faire ses courses au Walmart - bien mieux achalandé au nord de la linea - la frontière. Il concerne peut-être $10 \%$ de la population, et ne peut être obtenu que si les agents consulaires étasuniens sont convaincus que son obtention ne donnera pas lieu à une migration de travail. Il faut donc au moins attester d'une résidence mexicaine en propriété, d'un contrat de travail avec un salaire confortable, etc. Le simple visa touristique constitue le dernier niveau d'accès aux ressources des États-Unis, il est octroyé avec d'autant plus de parcimonie qu'il fonctionne bien souvent comme une première marche vers le marché de l'emploi américain.

L'attractivité de la zone frontalière repose largement sur la proximité de ces ressources étasuniennes (santé, éducation, biens de consommation courantes etc.), qu'elles soient accessibles ou non: les emplois offerts par les maquiladoras sont, au moins en partie, occupés par des migrants internes, candidats à la traversée.

Illustration 3 - Le parc industriel d'Agua Prieta

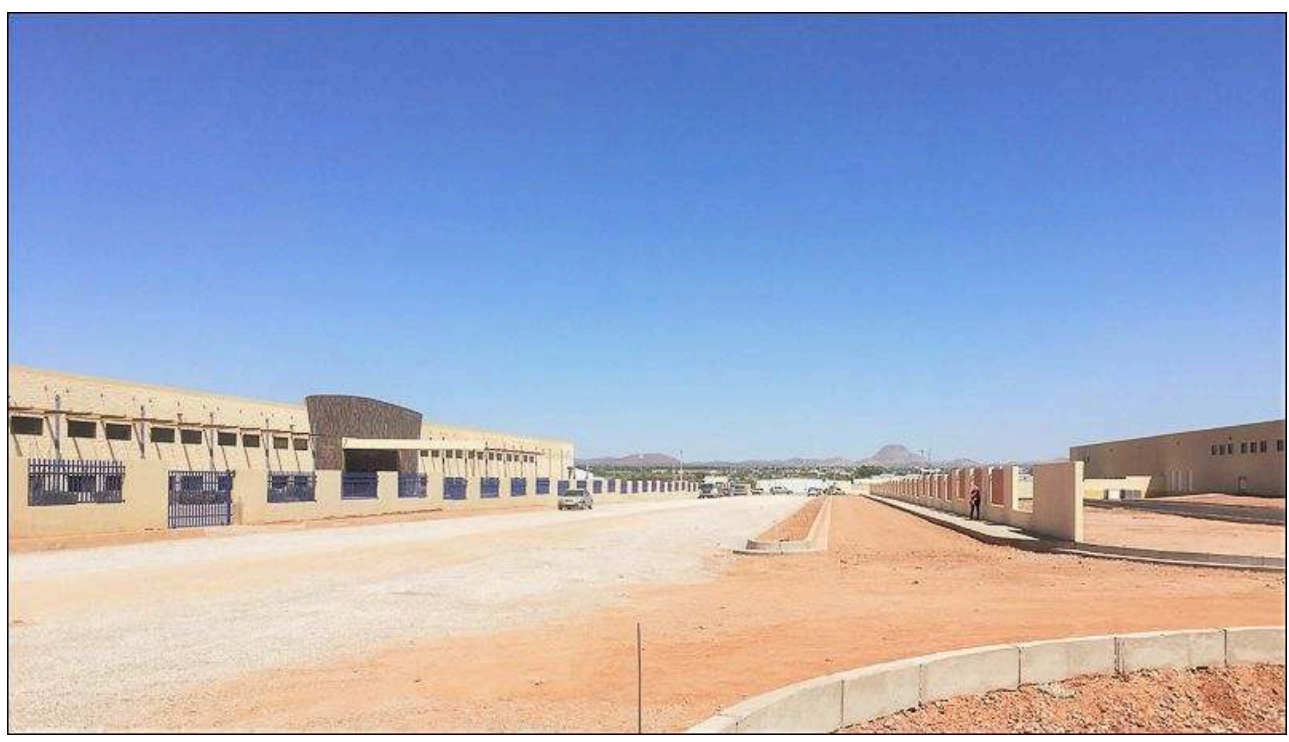

II se déploie à l'ouest du centre-ville, à quelques mètres de la frontière. Une fois reliées par la municipalité au service d'eau, d'électricité et au réseau asphalté, les parcelles sont louées, avec le bâtiment ou non, aux entreprises. La première usine du groupe CVG, productrice de composants électriques pour moteurs de poids lourds, à droite sur la photo, a dix ans. En face, une nouvelle usine vient de sortir de terre, pour ouvrir dans quelques semaines.

Auteurs : Aurélia Michel et Antonine Ribardière, 2017.

8 Le secteur automobile domine l'industrie à Agua Prieta : il concentre en 2013 près de la moitié des emplois industriels ${ }^{5}$; le secteur textile/ameublement est également bien représenté (près de $20 \%$ des emplois), ainsi que celui des composants électriques (près de $10 \%)$.

9 Les acteurs locaux estiment que l'industrie maquiladora représente aujourd'hui 14000 emplois, soit environ $70 \%$ de l'emploi formel de la ville. Ils s'accordent 
également pour souligner que plus de 1000 emplois sont vacants : la responsable des ressources humaines de l'usine CGV s'inquiète ainsi de ne pouvoir faire fonctionner sa nouvelle usine. L'entreprise a développé une politique de recrutement offensive : outre les annonces affichées sur les murs, diffusées à la radio ou par voitures haut-parleurs, des campagnes de recrutement "porte-à-porte" ont été mises en place dans les quartiers populaires de la ville. L'aire de recrutement a été largement étendue aux localités voisines, l'entreprise n'hésite pas à proposer des services de bus dans un rayon de 60 kilomètres: "J'irais bien les chercher jusque dans le Oaxaca, le Veracruz ou le Chiapas, mais nous n'avons pas de solution de logement à leur offrir ", explique la gérante.

\section{Illustration 4 - Arrivée à l'une des gares routières d'Agua Prieta}

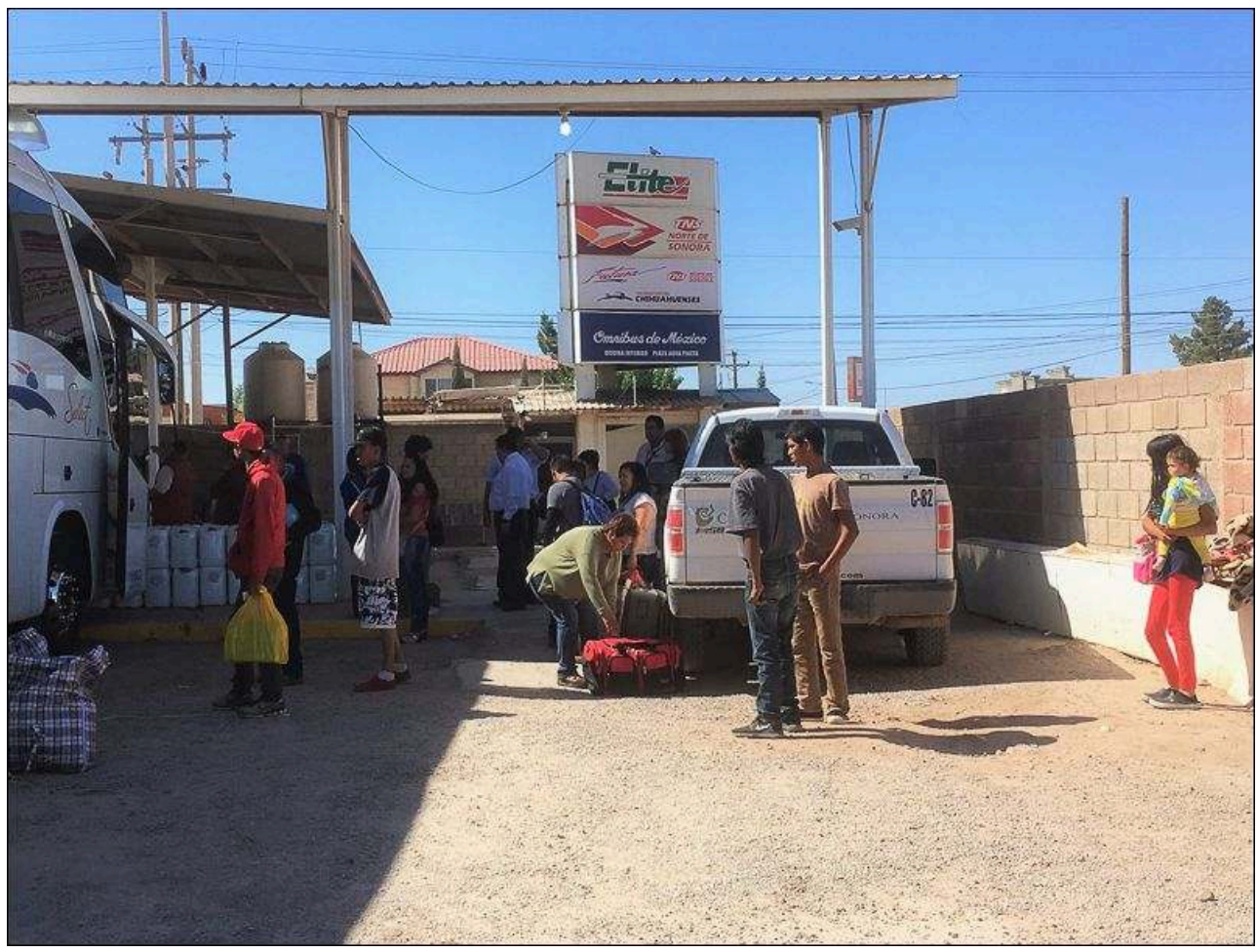

Les migrants du Sud du pays sont désormais moins nombreux.

Auteurs : Aurélia Michel et Antonine Ribardière, 2017.

En 2010, la majorité des résidents du municipe d'Agua Prieta étaient nés dans l'État du Sonora (77\%). Le poids des États voisins du Chihuahua et du Sinaloa atteste de l'importante des mouvements de population au sein de la région frontalière (respectivement 7,7 et 5,5\%). Ensuite, la contribution des migrations en provenance des États pauvres du Sud du Mexique apparaît nettement: en premier lieu le Chiapas, premier État de naissance en dehors de la Frontera, puis le Veracruz et enfin le Oaxaca.

11 Aujourd'hui, le temps des grandes vagues migratoires semble révolu. Les valeurs enregistrées à l'échelle de l'ensemble de la zone frontalière dans le cadre des enquêtes EMIF $^{6}$ rendent compte du tassement de l'apport migratoire : les arrivées en provenance du reste du pays déclinent en chiffres absolus à partir de 2010 et en valeurs relatives pour ce qui concerne les deux États du Chiapas et du Oaxaca. Les générations arrivées dans les années 1990, principalement de jeunes actifs, sont à l'origine d'une croissance naturelle particulièrement vive, qui alimente désormais la croissance urbaine. 
Aujourd'hui, les employés des maquiladoras sont en grande partie les enfants, voire les petits enfants, de la première génération de migrants.

Illustration 5 - Travail à la chaîne dans une fabrique de ceintures de sécurité pour le secteur automobile

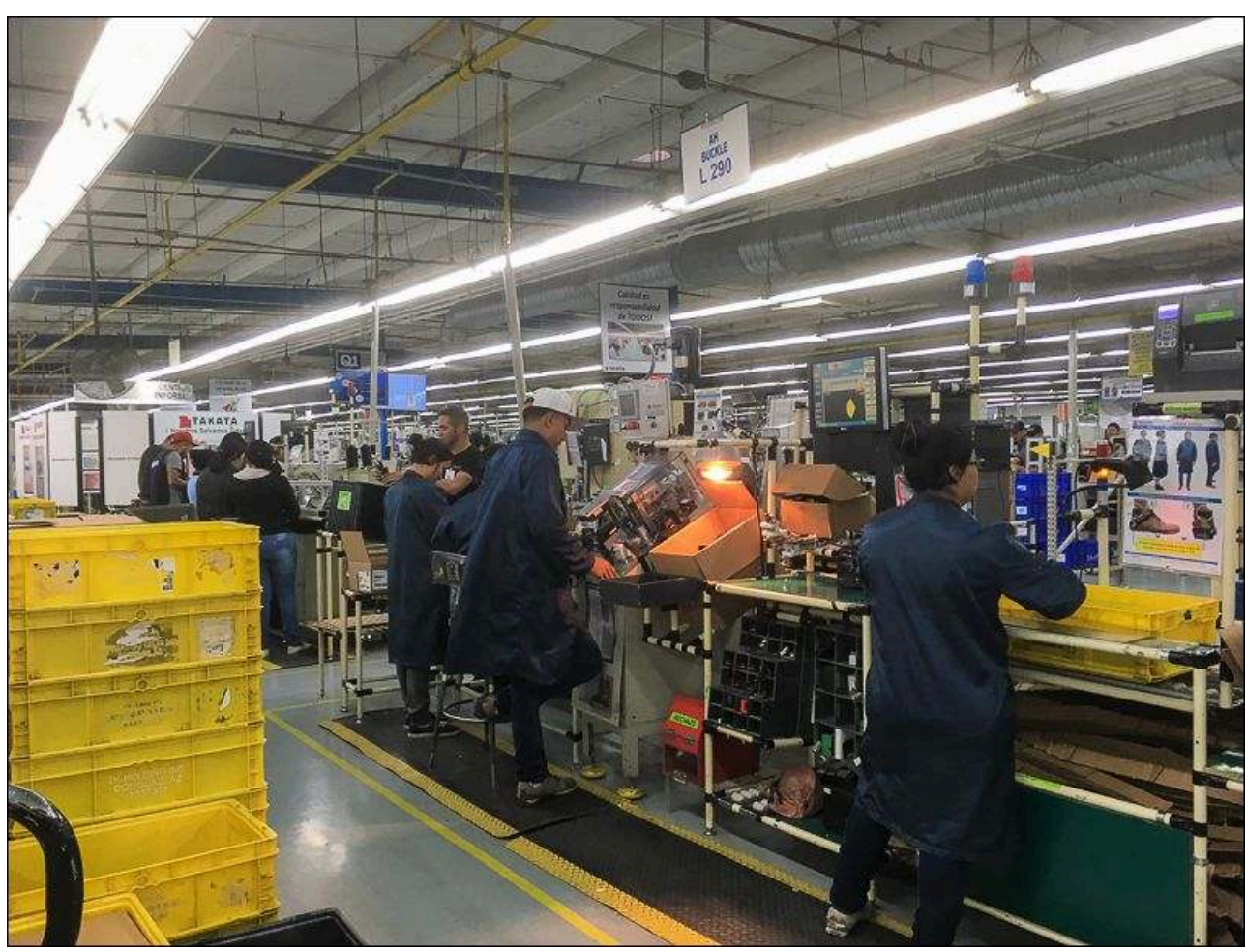

Auteurs : Aurélia Michel et Antonie Ribardière, 2017.

La plupart des usines fonctionnent 7 jours sur 7 , sur 2 tours : le premier commence à $6 \mathrm{~h}$, le deuxième se termine à minuit. Ces horaires d'embauche et de débauche rythment le fonctionnement de la ville tout entière. Les conditions de travail difficiles station debout, travail de précision qui occasionne des fatigues oculaires - contribuent à expliquer le turn-over très important. Les entreprises cherchent à retenir leurs employés en complétant les salaires très modestes par des primes - prime de présence, prime de ponctualité, prime de productivité, ou autres beneficios évoqués dans l'illustration 2 - qu'elles menacent de supprimer aux premières heures d'absence. Le principe du bas salaire, à la base du système, n'est jamais remis en cause, pas même par les syndicats. Toutefois l'emploi formel dans les maquiladoras est assorti d'un ensemble de prestations qui sont fixées par la loi fédérale de travail, en premier lieu la protection sociale (accès aux soins, à la retraite, également à un mode de garde) et l'accès au crédit immobilier (par l'organisme fédéral INFONAVIT qui permet d'accéder au crédit bancaire). Les entreprises prennent également à leur charge un certain nombre de prestations qui se substituent, pour une part, aux services publics ou aux politiques sociales fédérales, comme le transport, des prestations de santé spécifiques (lunettes à prix réduit par exemple), la prise en charge de certains frais alimentaires ou énergétiques sous la forme de bons. 
Illustration 6 - Entrée de l'usine CVG

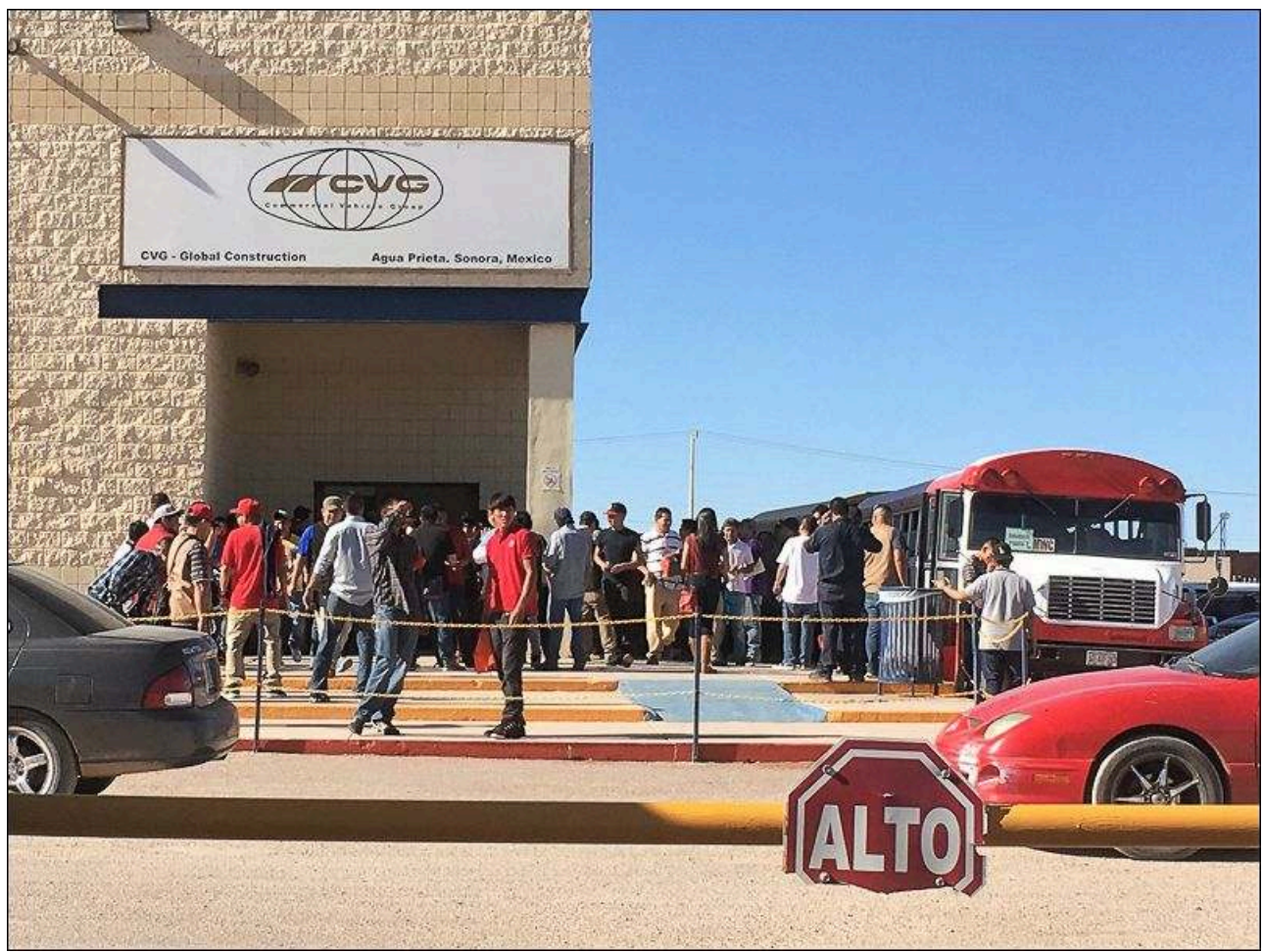

À l'heure de la débauche à CVG, des bus de l'entreprise reconduisent les employés non motorisés dans leurs quartiers de résidence, quelle que soit la localisation dans la ville. Un vaste parking, hors champ, permet également le stationnement des employés motorisés. Si l'entreprise est ouverte sur la ville, l'entrée est néanmoins contrôlée par un vigile - on distingue la barrière au premier plan.

Auteurs : Aurélia Michel et Antonie Ribardière, 2017.

Les employés sont jeunes : la DRH nous indique une moyenne d'âge de l'ordre de 25 ans dans cette entreprise, dans une proportion égale d'hommes et de femmes. Seul le niveau primaire est requis pour un poste d'opérateur ; la maîtrise de l'anglais constitue un réel atout pour progresser dans l'entreprise, ce qui pourrait constituer une opportunité pour les deportados - Mexicains reconduits à la frontière ces dernières années, qui se font parfois embaucher. Depuis 1997, la ville d'Agua Prieta compte un Instituto Tecnológico, qui propose des cursus supérieurs techniques et de gestion, et forme la plupart des cadres et employés qualifiés de la ville. 
Illustration 7 - Arrivée des bus de l'entreprise à la garderie

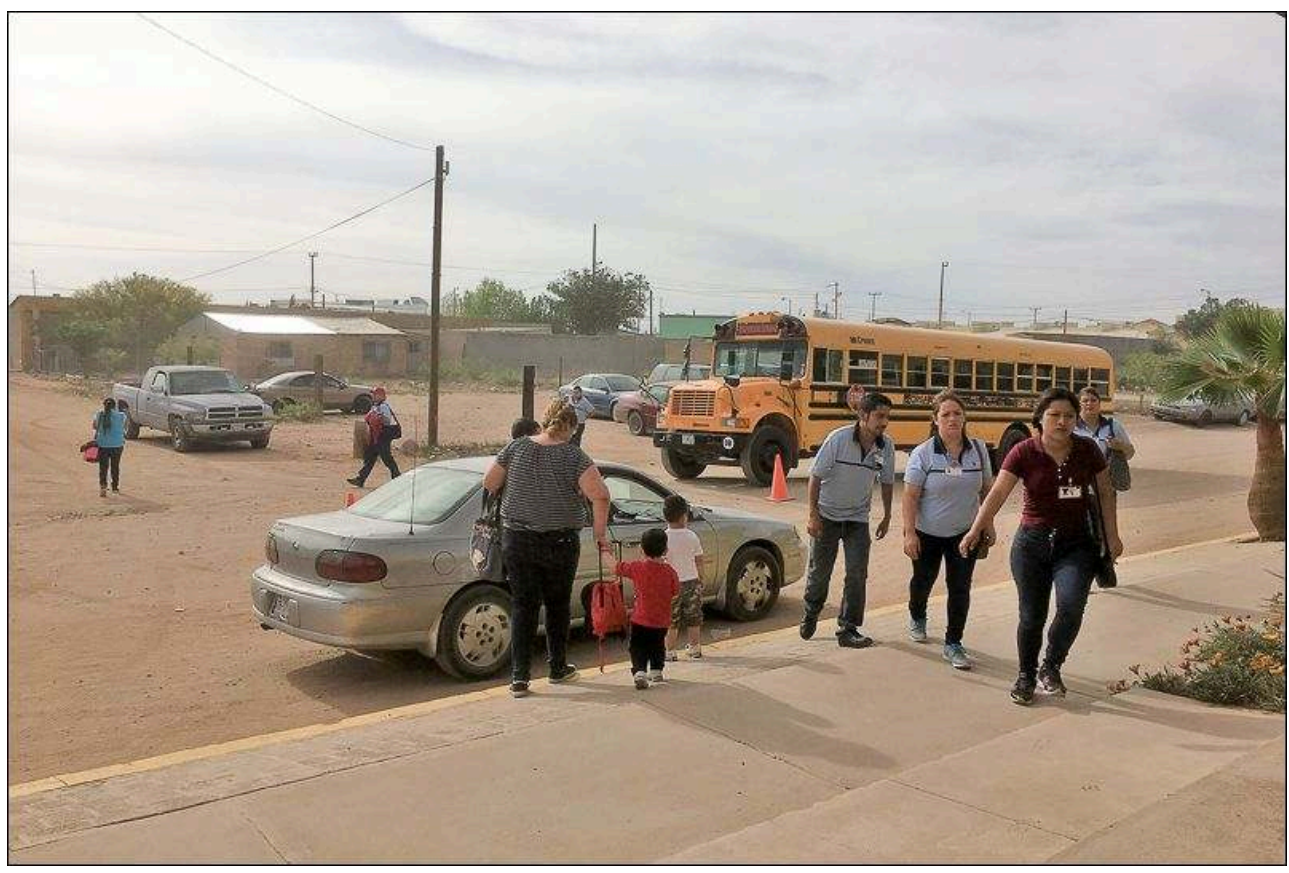

Les employés des maquiladoras ont encore le tee-shirt aux couleurs de l'entreprise.

Auteurs : Aurélia Michel et Antonine Ribardière, 2017.

14 La ville compte quatre garderies affiliées à la Sécurité sociale (Seguro social) qui accueillent, entre autres, les enfants des employés des maquiladoras. Les horaires sont adaptés au rythme du premier tour d'usine : ouverture dès $5 \mathrm{~h} 45$, fermeture à $16 \mathrm{~h} 45$; les bus des entreprises assurent les trajets de la garderie à l'usine. En raison des très bas salaires et du coût du logement, il est indispensable de cumuler au moins deux salaires par ménage. Le travail des mères est donc la règle, si possible dès la fin du congé de maternité - 6 semaines après la naissance. 
Illustration 8 - Fraccionamentos du programme INFONAVIT

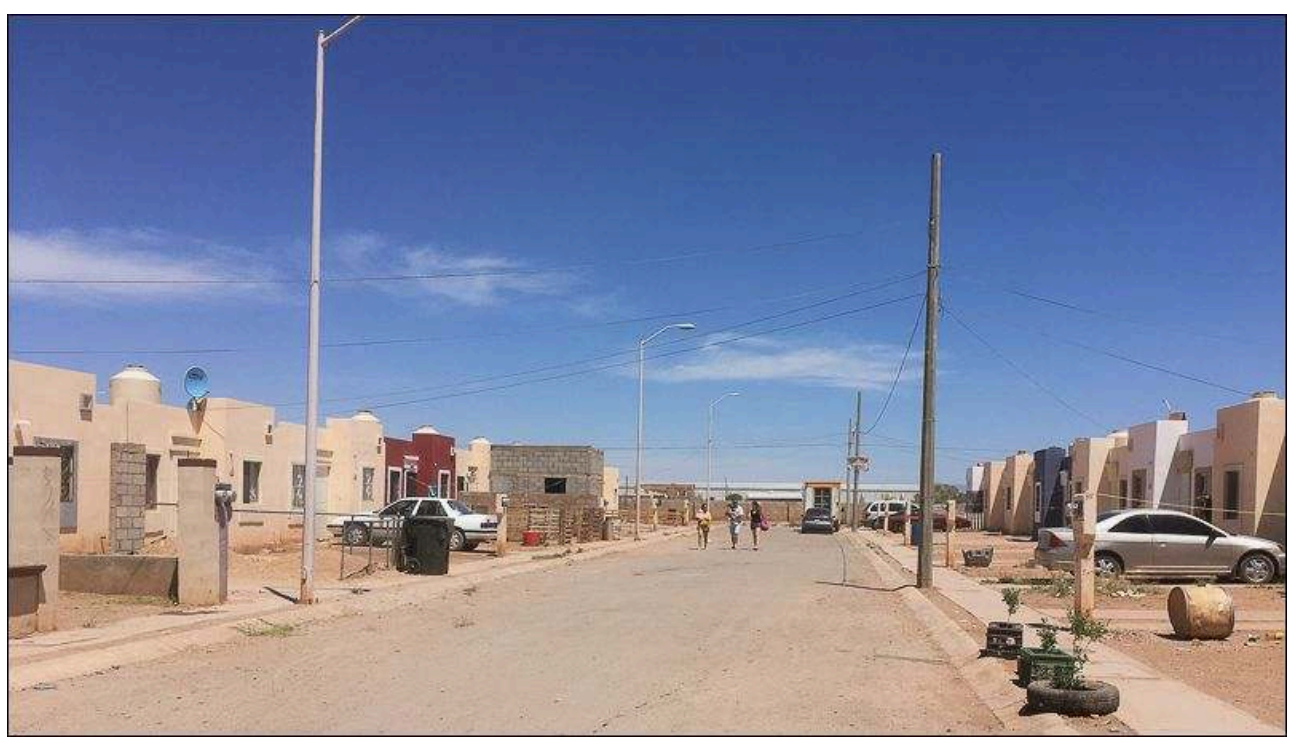

À deux pas de la zone industrielle ont été construits plusieurs fraccionamentos du programme INFONAVIT, lotissements composés de logements en accession à la propriété aidée. L'espace public est réduit au minimum, la proximité des logements est très forte.

Auteurs : Aurélia Michel et Antonine Ribardière, 2017.

Après deux ans d'ancienneté, l'employé qui cotise au Seguro Social peut bénéficier du programme fédéral INFONAVIT (Institut du Fonds National pour le Logement des Travailleurs). À Agua Prieta, moyennant un crédit payé environ 300 pesos par semaine durant vingt ans ( 16 dollars, un tiers du salaire de base environ), on peut devenir propriétaire d'une petite maison individuelle de $28 \mathrm{~m}^{2}$ (avec une seule chambre). Progressivement, les terrains libres devant les maisons sont occupés par une pièce supplémentaire auto-construite (cf. en position centrale de la photographie), qui peut être offerte à la location.

Depuis une douzaine d'années, trois lotissements de plusieurs centaines de logements chacun, ont été construits par des promoteurs privés à proximité du parc industriel. Un supermarché discount a été établi à proximité, et la municipalité y a implanté un hôpital et un parc d'attraction; prochainement, un centre d'accueil de jour pour les personnes âgées, financé par l'État du Sonora, devrait ouvrir, permettant ainsi aux actifs en charge d'un parent âgé de travailler.

Ces lotissements pour travailleurs du secteur formel et ces équipements sont orientés par les acteurs politiques et économiques locaux pour permettre le travail dans les maquiladoras. Situés dans la périphérie ouest de la ville, ils ne constituent cependant qu'une forme minoritaire du tissu urbain ; le plus grand nombre des actifs n'a pas accès à ces formes de crédits et/ou se contente de partager une chambre en colocation. 


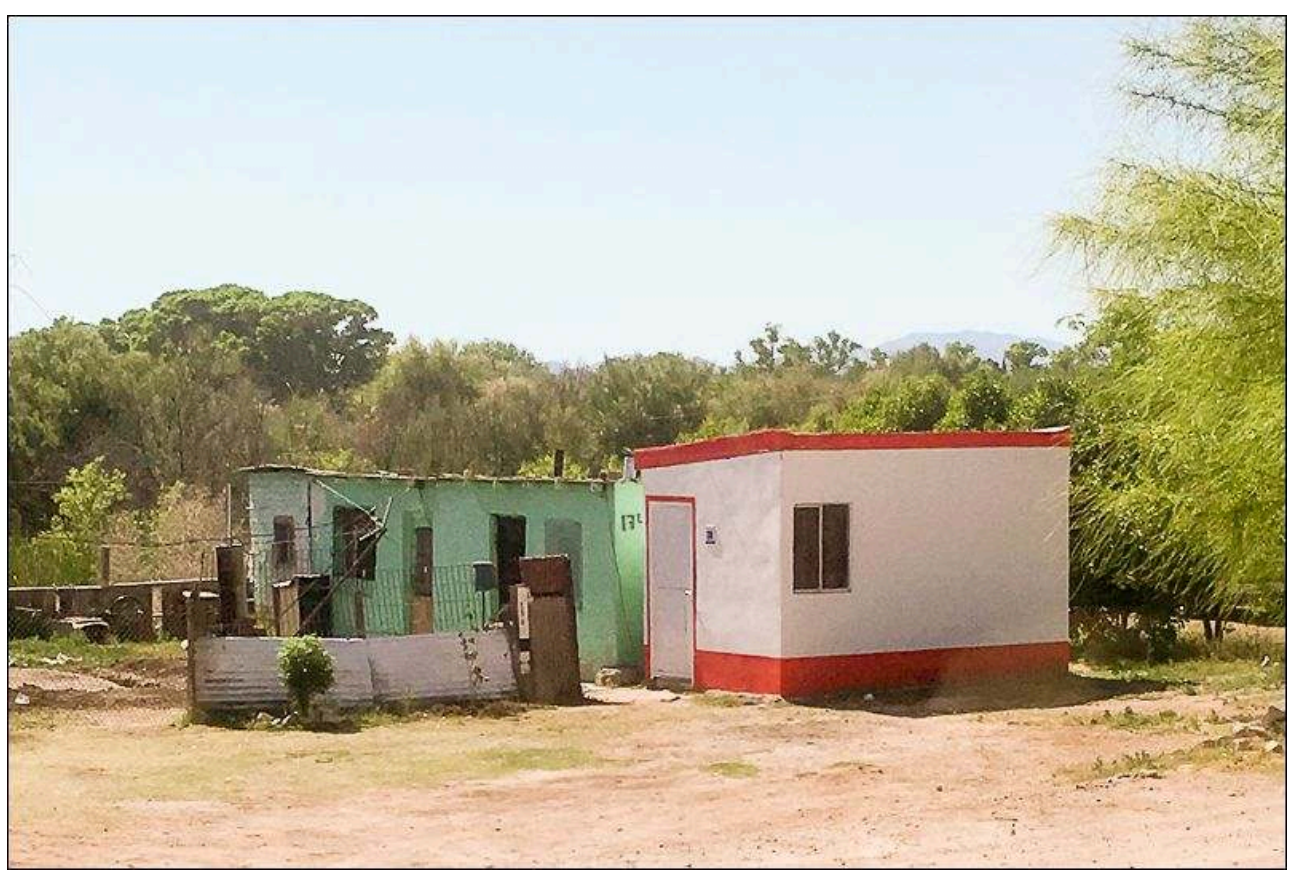

Une « casa rosa » est une maison fournie par le programme social de logement de l'État du Sonora, dans le quartier Ferrocarrril d'Agua Prieta.

Auteurs : Aurélia Michel et Antonine Ribardière, 2017.

Les zones résidentielles généralement autoconstruites, qui s'étendent en tache d'huile à partir du damier du centre-ville représentent la forme urbaine majoritaire à Agua Prieta. Emilia, qui est arrivée du Oaxaca il y a 20 ans, a construit sa maison dans l'actuelle rue 27, à l'époque encore inoccupée. Désormais, la ville compte une rue 52 où se trouvent les dernières parcelles urbanisées. Tous ces terrains appartiennent à l'ejido ; à ce jour, les habitants ne peuvent attester de titres de propriété en règle - attestation qui pourrait pourtant faciliter l'obtention d'un visa touristique, nécessaire pour accéder aux ressources de Douglas, voire aux emplois de l'Arizona. La municipalité a fourni l'eau et l'électricité, mais le drainage est encore inexistant et les eaux polluées du Rio Agua Prieta, dans lesquelles se déversent les ordures comme les eaux usées, jusqu'aux déchets toxiques de l'usine textile voisine, constituent un facteur de nuisance particulièrement agressif.

Une partie des habitants, en particulier les nouveaux venus et les jeunes ménages, ne peuvent encore - ou ne souhaitent - investir dans l'achat informel d'un terrain (20 000 pesos environ) et louent leur logement. Une chambre coûte environ 1500 à 2000 pesos mensuels, ce qui implique de la partager à plusieurs. Dans ce tissu hétérogène, mais dominé par l'auto-construction, quelques minuscules casas rosas (maisons roses), comme les désignent les habitants, constituent la contribution de l'État du Sonora à la question du logement (illustration 9). 
Illustration 10 - Le mur américain, un samedi après-midi près de la « linea »

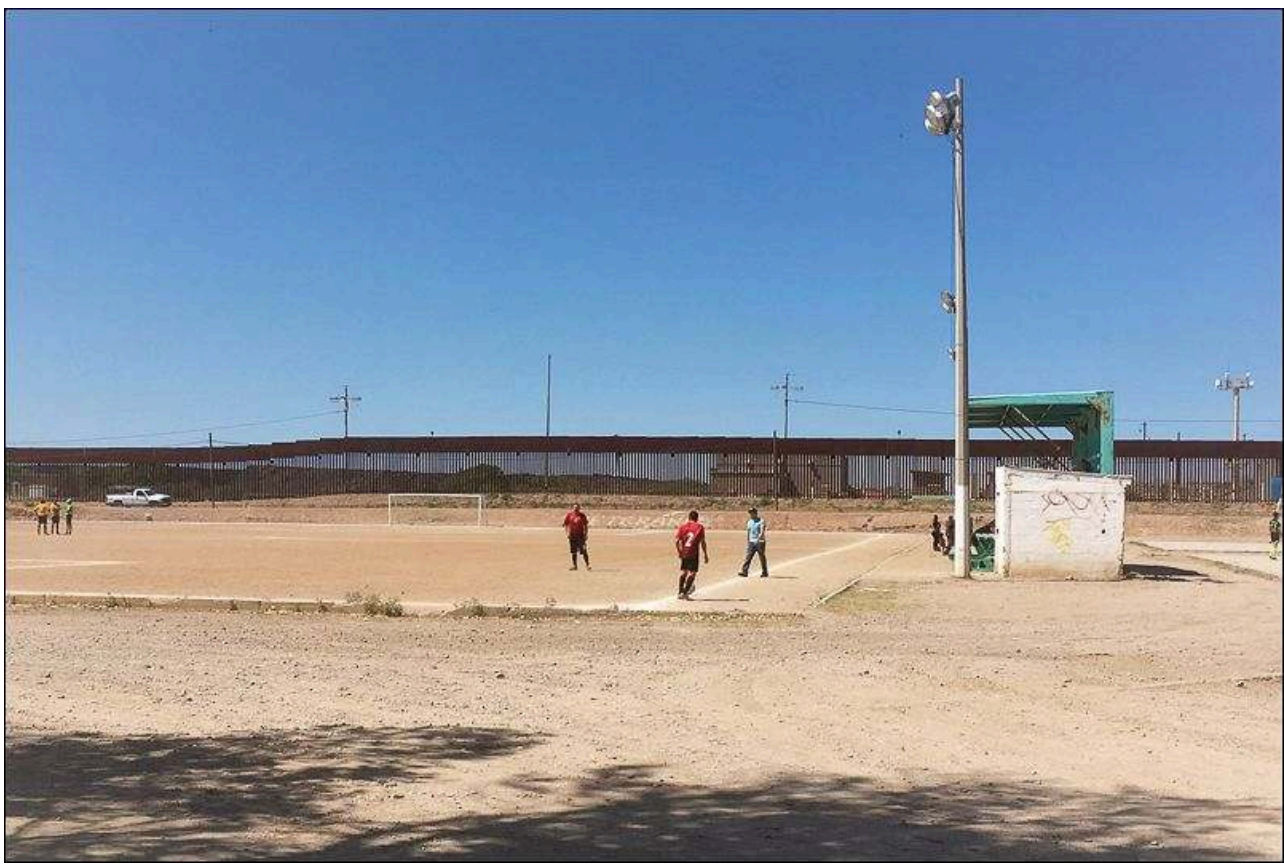

Constitué de lamelles métalliques, ce mur court depuis Tijuana, seulement interrompu au cœur du désert.

Auteurs : Aurélia Michel et Antonine Ribardière, 2017.

Face à la très forte croissance d'Agua Prieta ces quinze dernières années, les gouvernements municipaux comme de l'État du Sonora n'ont pas été en mesure d'adapter leurs services et de suivre le rythme de l'équipement nécessaire (voirie, drainage, traitement des eaux). Le principe même de la maquiladora, qui repose sur le maintien de bas salaires, reste déterminant dans l'accès à des ressources par définition limitées. Cependant le processus d'urbanisation se poursuit, avec les opportunités de ressources que cela implique: la présence de l'IUT, qui permet à certains de saisir d'autres opportunités professionnelles, la consolidation de l'habitat précaire et l'accès à la propriété, la protection sociale liée aux emplois, et puis, toujours en ligne de mire, la frontière et ses promesses...

21 Ces opportunités, construites peu à peu sur ce site désertique au seul argument de la frontière, constituent effectivement des ressources urbaines liées à la concentration spatiale (marché de l'emploi, offre diversifiée de services et de commerce, etc.), réparties dans l'espace en fonction de la distance à la frontière. Dans une certaine mesure, Agua Prieta incarne en miniature les hiérarchies et fragmentations sociales violentes du Mexique, accentuées par l'économie globale et la dépendance du pays vis à vis de son voisin : les plus proches de la linea, les plus ancrés, tirent un excellent parti de cette configuration spatiale, tandis qu'à mesure qu'on s'éloigne de cette ligne, les habitants sont de plus en plus vulnérables. En lisière de la ville, ils cumulent faibles ressources, informalité du statut du logement, absence des services basiques et exposition aux risques sanitaires dus à la pollution. Entre ces deux extrêmes, des classes moyennes ont pu émerger grâce à l'économie considérable générée par la maquiladora, qui exploite la faiblesse des salaires de la majorité. 


\section{BIBLIOGRAPHY}

Bizberg I., 2015. Le Mexique dans l'ALENA : intégration, dépendance et subordination économique. Politique américaine, 25, (1), p. 37-52.

Michel A., Ribardière A., 2017. Identifier les ressources urbaines pour lire les inégalités sociospatiales. EchoGéo [En ligne], 39 | 2017, mis en ligne le 28 mars 2017, consulté le 07 juin 2017. URL : http://echogeo.revues.org/14925 ; DOI : 10.4000/echogeo.14925

Musset A., 2010. Le Mexique. Presses Universitaires de France, collection Que sais-je?, 128 p.

Villavicencio D., 2004. Les « Maquiladoras » de la frontière nord du Mexique et la création de réseaux binationaux d'innovation. Innovations, vol. 19, $\mathrm{n}^{\circ}$ 1, p. 143-161.

\section{NOTES}

1. Pour une discussion des relations récentes Mexique/États-Unis, voir Bizberg, 2015.

2. Pour une présentation des maquiladoras et des évolutions qu'elles ont connues sur la frontière Nord, voir Villavicencio, 2004. Pour une mise en perspective des maquiladoras dans le contexte mexicain, voir Musset, 2004.

3. Cet article s'appuie sur une mission de terrain réalisée en avril 2017, au cours de laquelle une série d'entretiens ont été réalisés auprès (i) de responsables politiques et administratifs locaux, (ii) de responsables de ressources humaines, de représentants syndicaux et d'employés de maquiladoras et (iii) de personnes originaires du Oaxaca, pour l'essentiel. Cette mission a été réalisée dans le cadre du PICS RESUM, financé par le CNRS. Nous remercions ici en particulier Nahum, Pablo et Eleazar pour leur aide précieuse lors de l'enquête.

4. Dotation collective de terres par l'État qui fut le principe de la réforme agraire mise en place par la Révolution mexicaine à partir de 1917. L'ejido d'Agua Prieta a été créé en 1921, octroyant 10000 hectares inaliénables à 114 bénéficiaires.

5. Sources : Censo Economico 2014, INEGI.

6. Enquêtes sur la migration à la frontière nord (Encuesta sobre Migración en la Frontera Norte de México (Emif Norte)), réalisées par le COLEF depuis 1995. 